\title{
Adenocarcinoma Originating From a Completely Isolated Duplication Cyst of the Mesentery in an Adult
}

\author{
Seung Yong Shin, Mee Yon Cho ${ }^{1}$, Hoon Ryu' ${ }^{2}$ Jae Woo Kim, Hyun-Soo Kim, Jung Min Kim, An Na Ko, \\ Tae-Sun Yu, Hong Jun Park \\ Department of Internal Medicine, Pathology ${ }^{1}$, and General Surgery', Yonsei University Wonju College of Medicine, Wonju, Korea
}

\begin{abstract}
Alimentary tract duplications are uncommon congenital abnormalities that usually have an anatomical connection with some part of the gastrointestinal tract and have a common blood supply with the adjacent segment of intestine. A completely isolated duplication cyst (CIDC) is a very rare type of gastrointestinal duplication that does not communicate with the normal bowel segment and possesses its own exclusive blood supply. Only 5 CIDC cases in adults have been reported in the English medical literature. Additionally, only 1 case of mucinous cystadenoma from an infected CIDC of the ileum has been reported. This report describes a 52-year-old male patient with a peritoneal CIDC, which upon curative excision was found to have given rise to an adenocarcinoma. The latter was lined internally with malignant glandular cells and contained a smooth muscular outer layer as determined by microscopic examination of the tissue. We believe that this is the first reported case of an adenocarcinoma originating from a CIDC in an adult. (Intest Res 2014;12:328-332)
\end{abstract}

Key Words: Adenocarcinoma; Completely isolated duplication cyst; Duplication cyst; Enteric duplications; Enterogenous cyst

\section{INTRODUCTION}

Duplication cysts are rare congenital abnormalities that are usually diagnosed in childhood, and the occurrence of such cysts is rare in adulthood. ${ }^{1,2}$ The cysts can occur anywhere from the mouth to the anus. Duplication cysts have been described as congenital malformations that involve the mesenteric side of the gastrointestinal tract and share a common wall or blood supply with the involved bowel. ${ }^{3}$

Most cases are diagnosed during early childhood because duplication cysts tend to accompany symptoms such as abdominal pain or palpable masses in this age group. In contrast, these cysts are diagnosed incidentally as asymptomatic disease during adulthood. There are several associated com-

Received September 22, 2013. Revised October 27, 2013.

Accepted October 28, 2013.

Correspondence to Hong Jun Park, Department of Internal Medicine, Yonsei University Wonju College of Medicine, 20 Ilsan-ro, Wonju 220-701, Korea.

Tel: +82-33-741-1226, Fax:+82-33-741-1228,E-mail: ppakongs@daum. net

Financial support: None. Conflict of interest: None. plications, including bleeding, fistula formation, and inflammatory masses. ${ }^{4,5}$ Furthermore, malignant transformation can occur, although this is rare ${ }^{6,7}$ These complications might be caused by heterotropic mucosa of a gastric or pancreatic origin, which is a common finding in histological examinations of duplication cysts. ${ }^{8}$

Generally, duplication cysts are firmly attached to the digestive tract and are most frequently found around the ileocecal region. Thus, it is essential to remove the bowel segment containing the duplication cyst when surgical intervention is necessary. ${ }^{9}$ Completely isolated duplication cysts (CIDCs) comprise an extremely rare type of gastrointestinal duplications, which possess their own blood supply and do not communicate with the normal bowel segment. ${ }^{10,11}$

Only 5 CIDCs in adults were reported in the English medical literature, ${ }^{10-14}$ and only 1 case of a mucinous cystadenoma that originated in an infected ileal CIDC has been reported, in 2012. ${ }^{10}$ However, to date, there are no reports of adenocarcinoma arising from a CIDC. We present the first case of an adenocarcinoma originating from a CIDC in an adult who

๑ Copyright 2014. Korean Association for the Study of Intestinal Diseases. All rights reserved.

This is an Open Access article distributed under the terms of the Creative Commons Attribution Non-Commercial License (http://creativecommons.org/licenses/by-nc/3.0)

which permits unrestricted non-commercial use, distribution, and reproduction in any medium, provided the original work is properly cited. 
underwent a curative mass excision, along with a literature review.

\section{CASE REPORT}

A 52-year-old male patient with abdominal pain was admitted to the Wonju Severance Christian Hospital. His medical history was unremarkable, except for pulmonary tuberculosis, which had been cured with medication 6 months earlier. An intraperitoneal mass was noted on abdominopelvic CT conducted by an external facility (Fig. 1A).

The patient had mild abdominal pain in the middle of his abdomen that was not associated with food intake or positional change. Upon system review, there were no specific findings such as weight loss. Findings of the physical examination were unremarkable and did not reveal a palpable mass or abdominal tenderness. Laboratory analysis revealed a CEA level of $29.3 \mathrm{ng} / \mathrm{mL}$ (standard value: $<5 \mathrm{ng} / \mathrm{mL}$ ), and a markedly increased level of CA 19-9 of up to $4881 \mathrm{U} / \mathrm{mL}$ (standard value: $<5 \mathrm{U} / \mathrm{mL}$ ). Other laboratory results were within normal ranges.

Chest CT revealed improved pulmonary tuberculosis and an approximately 4.3 -cm-sized cystic mass in the sub-gastric area; the internal contents of the mass showed an enhanced density greater than that of the surrounding fluid. The mass contained a partially calcified region and was not attached to an adjacent organ such as the stomach or small intestine.

The endoscopy finding from an external facility indicated only chronic superficial gastritis. There was no visible mass or mass effect lesion. Endoscopic ultrasound (EUS) was available for further evaluation of the mass because it was located near the lower border of the stomach. On EUS, the
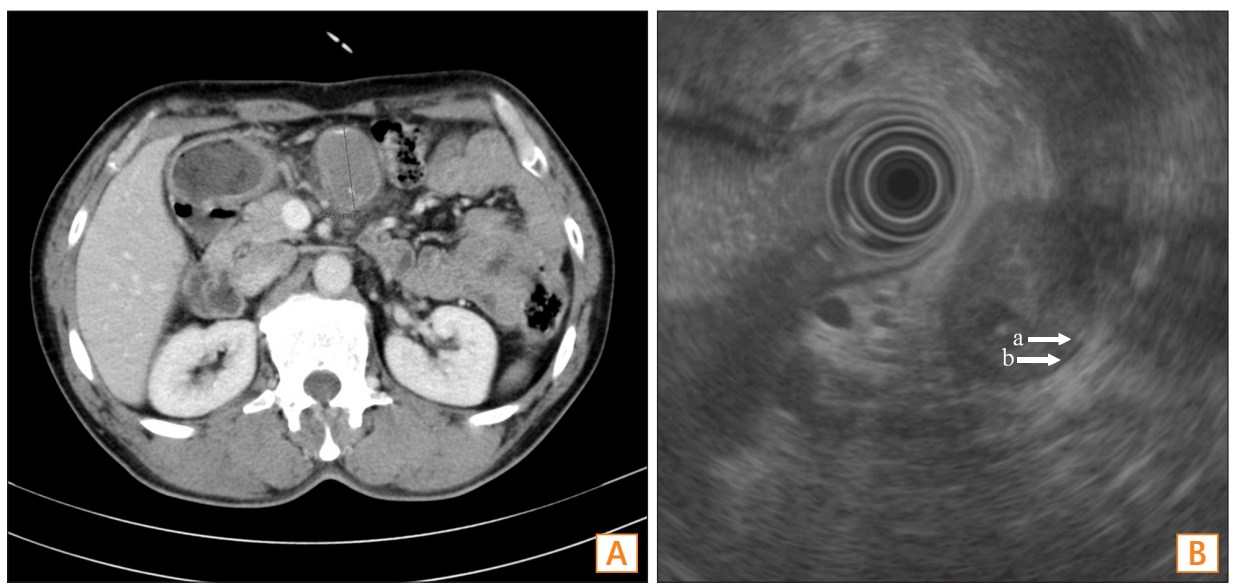

Fig. 1. Abdominopelvic CT and EUS findings. (A) An abdominopelvic CT from an external facility showed an intraperitoneal mass near the stomach. (B) EUS revealed that the mass was in an extragastric area and had cystic features with an internal mixed echoic pattern. Furthermore, the cystic mass wall had an inner smooth hyperechoic mucosal layer (arrow a) and an outer hypoechoic muscular layer (arrow b).
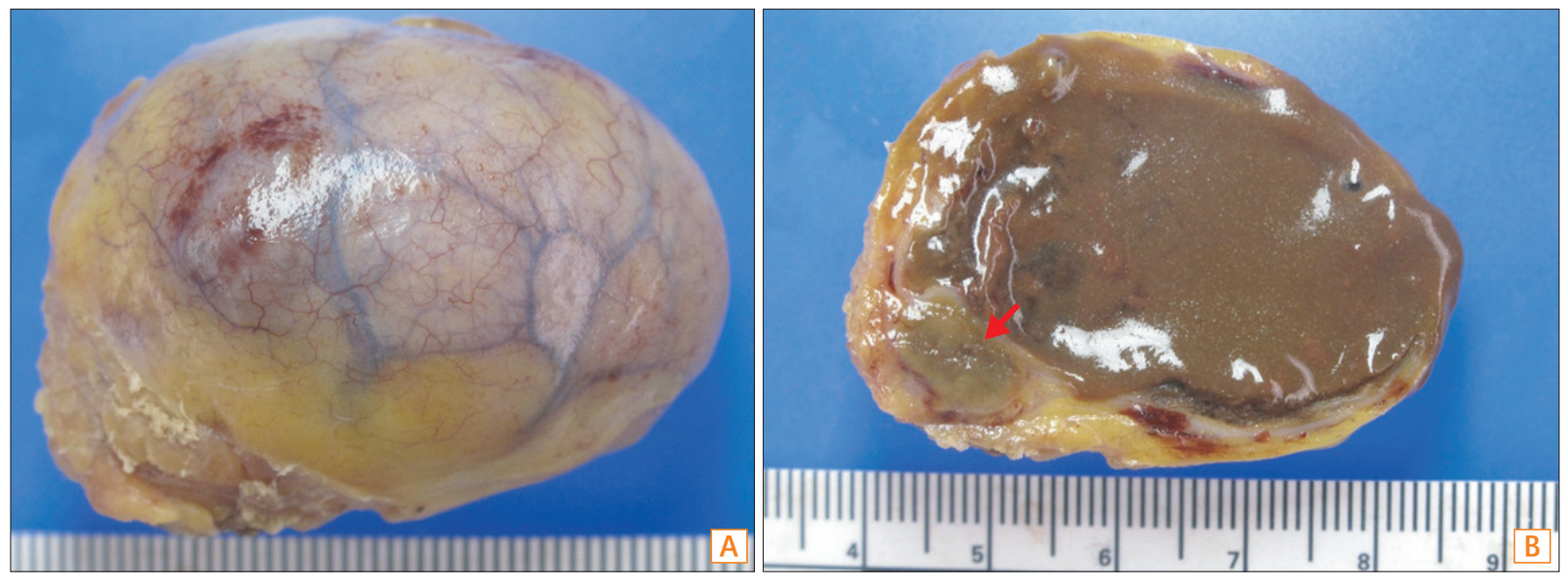

Fig. 2. Gross finding of removed cyst (A), and feature of gross section (B). The entire cyst was excised without disturbing the normal bowel or mesenteric anatomy (A). In a gross section analysis, it was determined to be a $4 \times 3 \times 3-\mathrm{cm}-$ sized unilocular cyst filled with dark brown necrotic material that appeared to comprise hemorrhagic contents. The cyst wall was evenly thin with a focal, ill-defined, yellowish-brown mural nodule (red arrow) (B). 
mass was found to be in the extra-gastric area and presented as a cystic feature with an internally mixed echoic pattern, and the cystic mass wall had both an inner smooth hyperechoic mucosal layer (Fig. 1B, arrow a) and an outer hypoechoic muscular layer (Fig. 1B, arrow b).

On the basis of these findings, we diagnosed this case as a malignant gastrointestinal tumor or malignant lymph node and performed surgery. The entire cyst was excised without disturbing the normal bowel or mesenteric anatomy. The mass featured a well-encapsulated oval shape (Fig. 2A). During surgery, we found that the mass was located in the peritoneum, was not attached to the small bowel or stomach, and had a separate feeding vessel. Upon a gross sectional inspection, the mass was a $4 \times 3 \times 3$-cm unilocular cyst filled with dark brown necrotic material that appeared to comprise hemorrhagic contents. The cyst wall was evenly thin with focally located, ill-defined yellowish-brown mural nodules (Fig. 2B). Upon microscopic examination, the cyst wall was found to be composed of an inner columnar epithelial lining, 2 smooth muscle layers, and serosa, thus mimicking the intestine. However, the epithelial lining of the entire cyst consisted of gland-forming neoplastic columnar epithelium, which was characterized by a loss of nuclear polarity, a high nuclear/cytoplasmic ratio, and hyperchromasia, without spared non-neoplastic epithelium (Fig. 3A). Focal areas of invasion into the smooth muscle were observed, and the
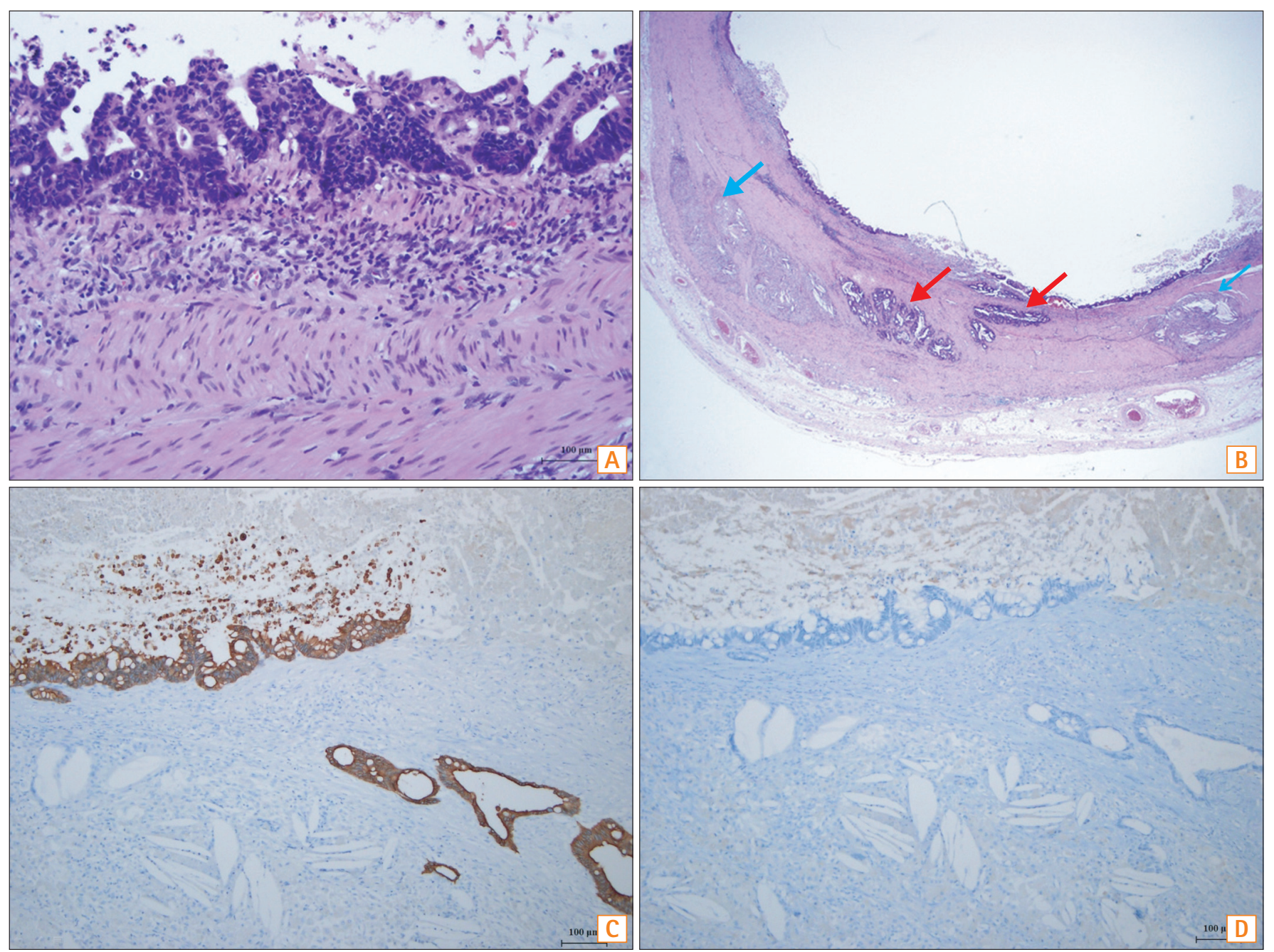

Fig. 3. The cyst wall epithelial lining. The inner surface of the cyst was lined with neoplastic columnar epithelium that exhibited an increased nuclearl cytoplasmic ratio with a loss of polarity and nuclear hyperchromasia. Non-neoplastic epithelium was not observed in the cyst. The smooth muscle layers of the cyst resembled those of the intestinal wall $(\mathrm{HEtE}, \times 20)(A)$. The neoplastic glandular epithelium was observed to focally invade the muscle layer (red arrow) (B). There were multifocal cholesterol granulomas in the wall of the cyst, 1 of which matched the yellowish nodule observed during the gross examination (blue arrow; HEE $\times$ $\times 1.25)$. Immunohistochemical $(\mathrm{IHC})$ staining. IHC staining of the neoplastic epithelial lining of the cyst was positive for cytokeratin 20 (C) and negative for cytokeratin 7 (D) $(\mathrm{HEE}, \times 100)$. 
cyst wall contained multifocal cholesterol granulomas that matched the yellowish nodules observed during the gross examination (Fig. 3B). The immunohistochemical stains were positive for cytokeratin 20 and negative for cytokeratin 7 in the neoplastic epithelial lining of the cyst (Fig. 3C, D). These findings were consistent with an adenocarcinoma that had arisen from the intestinal duplication cyst.

There were no metastases or local cancer invasion, according to $18 \mathrm{~F}$-fluorodeoxyglucose PET-CT. No postoperative complications occurred, the patient remains well, and the tumor marker levels had decreased to within normal ranges at follow-up.

\section{DISCUSSION}

Duplication cysts in the alimentary tract are rare developmental anomalies that involve the mesenteric side of the associated alimentary tract and share a common blood supply with the native bowel. ${ }^{2}$ These cysts can occur anywhere along the alimentary tract from the mouth to the anus, although the ileum is the most frequently involved region (35\%) followed by the esophagus (19\%), jejunum (10\%), stomach (9\%), and colon (7\%). ${ }^{15}$ CIDC is an extremely rare variant form of gastrointestinal duplication that has its own blood supply and does not communicate with the normal bowel segment.

To our knowledge, only 5 CIDC cases have been found in adults among 8 reported cases to date. ${ }^{10-14}$ Only 1 case of a malignant transformation from CIDC (mucinous cystadenoma) in an adult has been reported..$^{10}$ In the present case, during abdominal surgery, we diagnosed an adenocarcinoma that originated from a CIDC in a 52-year-old male with nonspecific abdominal pain. To our knowledge, this is the first report of such a case.

Several theories were proposed to account for the development of enteric duplications, including recanalization after the solid epithelial stage of embryonic bowel development, persistent embryologic diverticula, and the intrauterine vascular accident theory. ${ }^{15-17}$ Bremer's "aberrant vacuolization" theory suggests that epithelial proliferation occurs during embryonic intestine development and occludes the bowel lumen in the 6-week embryo ("solid stage"); thereafter, a vacuolization of the entire alimentary tract occurs, thus transforming the digestive system into a tube with a single lumen. Throughout the process of vacuole coalescence, an error might occur ("aberrant vacuolization"), resulting in the formation of 2 (or more) parallel channels that may or may not communicate with each other. ${ }^{8}$ However, no single theory adequately explains all known duplications. ${ }^{8}$

Malignant changes of in communicating type duplication cysts were most frequently observed in the small bowel, followed by the colon, rectum, and stomach. ${ }^{17,18}$ Such changes can originate from the underlying mucosa and develop into adenocarcinoma in almost all cases. However, in our case, there was no remaining normal mucosa, only heterotrophic mucosa. Once a malignant change occurs, the prognosis is generally poor because of the high metastasis rate and rare presentation of symptoms in adults. Therefore, early diagnosis and management are essential for duplication cysts.

Diagnoses of duplication cysts are generally made by abdominal CT, MRI, and ultrasonography ${ }^{19,20}$ Duplication cysts manifest as smooth, rounded, fluid-filled cysts or tubular structures with thin, slightly enhancing walls on CT scans. ultrasonography and MRI might indicate a duplication via the identification of a 3-layered image that represents the walled layer of the duplication cyst. Additionally, if a CIDC is located in an available area, EUS might be helpful because it can allow a detailed identification of the internal contents of cysts and discriminate the cyst wall layers. The pathologic characteristics of duplication cysts include spherical or tubular structures that are firmly attached to at least 1 point in the alimentary tract, possess well-developed smooth muscle layers, and have an epithelial lining that resembles some part of the alimentary tract.

In conclusion, we report herein a rare case of adenocarcinoma that originated from a CIDC in an adult. This case suggests that the various duplication cyst forms and the associated possibilities of malignant transformation should be considered for the early detection and treatment of uncommon duplication cysts.

\section{RFERENCES}

1. Johnson JA, 3rd, Poole GV. Ileal duplications in adults. Presentation and treatment. Arch Surg 1994;129:659-661.

2. Carachi R, Azmy A. Foregut duplications. Pediatr Surg Int 2002; 18:371-374.

3. Schalamon J, Schleef J, Hollwarth ME. Experience with gastrointestinal duplications in childhood. Langenbecks Arch Surg 2000;385:402-405.

4. Lim GY, Im SA, Chung JH. Complicated duplication cysts on the ileum presenting with a mesenteric inflammatory mass. Pediatr Radiol 2008;38:467-470.

5. Prabhu SM, Anand R, Narula MK, et al. Mesenteric cysts associated with recurrent small-bowel volvulus: cause or effect? Jpn J Radiol 2012;30:858-862. 
6. Shivnani AT, Small W, Jr., Benson A, 3rd, Rao S, Talamonti MS. Adenocarcinoma arising in rectal duplication cyst: case report and review of the literature. Am Surg 2004;70:1007-1009.

7. Stringer MD. Adenocarcinoma within a rectal duplication: case report and literature review. Ann R Coll Surg Engl 2000;82:146.

8. Olajide AR, Yisau AA, Abdulraseed NA, Kashim IO, Olaniyi AJ, Morohunfade AO. Gastrointestinal duplications: experience in seven children and a review of the literature. Saudi J Gastroenterol 2010;16:105-109.

9. Sinha A, Ojha S, Sarin YK. Completely isolated, noncontiguous duplication cyst. Eur J Pediatr Surg 2006;16:127-129.

10. Collaud S, Bayerl C, Wille G, et al. Mucinous cystadenoma arising in a completely isolated infected ileal duplication cyst. S Afr J Surg 2012;50:45-46.

11. Kyriakos N, Andreas C, Elena S, Charalampos A, Chrisanthos G. Infected completely isolated enteric duplication cyst management with percutaneous drainage and surgical excision after retreat of infection: a case report. Case Rep Surg 2013;2013:108126.

12. Gumus M, Kapan M, Gumus H, Onder A, Girgin S. Unusual noncommunicating isolated enteric duplication cyst in adults. Gastroenterol Res Pract 2011;2011:323919.

13. Kim SK, Lim HK, Lee SJ, Park CK. Completely isolated enteric duplication cyst: case report. Abdom Imaging 2003;28:12-14.

14. Pant N, Grover JK, Madan NK, Chadha R, Agarwal K, Choudhury SR. Completely isolated enteric duplication cyst associated with a classic enterogenous duplication cyst. J Indian Assoc Pediatr Surg 2012;17:68-70.

15. Ramakrishna HK. Intestinal duplication. Indian J Surg 2008;70: 270-273.

16. Blank G, Königsrainer A, Sipos B, Ladurner R. Adenocarcinoma arising in a cystic duplication of the small bowel: case report and review of literature. World J Surg Oncol 2012;10:55.

17. O'Donnell PL, Morrow JB, Fitzgerald TL. Adult gastric duplication cysts: a case report and review of literature. Am Surg 2005; 71:522-525.

18. Kim TH, Kim JK, Jang EH, Lee JH, Kim YB. Papillary adenocarcinoma arising in a tubular duplication of the jejunum. Br J Radiol 2010;83:e61-e64.

19. Segal SR, Sherman NH, Rosenberg HK, et al. Ultrasonographic features of gastrointestinal duplications. J Ultrasound Med 1994;13:863-870.

20. Tew K, Soans BK, Millar EA. Adenocarcinoma in an ileal duplication cyst: ultrasound and computed tomography findings. Australas Radiol 2000;44:228-231. 